\title{
Diclofenac Versus Ketorolac for Pain Control After Primary Total Joint Arthroplasty: A Comparative Analysis
}

Nicole E. George ${ }^{1}$, Cheryle Gurk-Turner ${ }^{2}$, Nequesha S. Mohamed ${ }^{3}$, Wayne A. Wilkie ${ }^{3}$, Ethan A. Remily ${ }^{3}$, Iciar M. Dávila Castrodad ${ }^{4}$, Elana Roadcloud ${ }^{3}$, Ronald Delanois ${ }^{3}$

1. Orthopedic Surgery, Aultman Hospital, Canton, USA 2. Pain Management, Lifebridge Health-Rubin Institute for Advanced Orthopedics, Baltimore, USA 3. Orthopedics, Lifebridge Health-Rubin Institute for Advanced Orthopedics, Baltimore, USA 4. Orthopedic Surgery, Hackensack Meridian School of Medicine at Seton Hall University, Nutley, USA

Corresponding author: Ronald Delanois, delanois@me.com

\section{Abstract \\ Introduction}

As total hip arthroplasty (THA) and total knee arthroplasty (TKA) transition to outpatient settings, appropriate pain management remains a challenge. Nonsteroidal anti-inflammatory drugs (NSAIDs) may subvert the need for postoperative opioids. This study evaluated: 1) total opioid consumption; 2) postoperative pain intensity; 3) discharge destination; 4) length of stay (LOS); and 5) THA and TKA patients' satisfaction in receiving adjunctive intravenous (IV) diclofenac or ketorolac.

\section{Methods}

In this retrospective cohort study, patients scheduled to undergo primary THA or TKA by a single surgeon between March 2017 and April 2018 were identified. Patients were stratified based on the receipt of IV diclofenac (THA: $\mathrm{n}=25$; TKA: $\mathrm{n}=51$ ) or IV ketorolac (THA: $\mathrm{n}=28$; TKA: $\mathrm{n}=32$ ) in addition to the standard pain management regimen. Student's t-testing and Chi-square were used to analyze continuous and categorical variables, respectively.

\section{Results}

TKA diclofenac patients had lower opioid consumption 12 hours postoperatively (p: 0.037). TKA patients in the diclofenac cohort were discharged to home less often (p: 0.025). Both diclofenac cohorts had greater patient satisfaction than the ketorolac cohorts (p: <0.05). There was no significant difference between groups in postoperative pain intensity at 24 or 48 hours or in the length of stay (p: $>0.05$ for all).

\section{Conclusion}

Received 02/06/2020 Review began 02/13/2020 Review ended 03/10/2020 Published 03/18/2020

() Copyright 2020 George et al. This is an open access article distributed under the terms of the Creative Commons Attribution License CC-BY 4.0., which permits unrestricted use, distribution, and reproduction in any medium, provided the original author and source are credited.
This study demonstrated that both TKA and THA patients treated with IV diclofenac had no difference in postoperative pain intensity while THA patients had no difference in opioid consumption relative to those treated with IV ketorolac. Further comparison of IV NSAIDs with other IV pain medications may provide broader insight into the ideal management for postoperative pain for this widening patient population.

Categories: Pain Management, Orthopedics

Keywords: diclofenac, ketorolac, total hip arthroplasty, total knee arthroplasty, pain control

\section{Introduction}

Total joint arthroplasty (TJA) poses a significant economic burden to the healthcare system, with combined inpatient costs for Medicare total hip and knee arthroplasties exceeding USD 7 billion in 2014 [1]. Innovations and improvements in total knee arthroplasty (TKA) and total hip arthroplasty (THA) have helped reduce the burden of postoperative care required for both and combat the rising cost of care $[2,3]$. As a result, TKA was removed from the list of inpatient-only procedures by the Centers for Medicare \& Medicaid Services (CMS) in 2018, with THA to follow in 2020 [4]. As the demand for TKA and THA continues to increase, the anticipated volume shift to outpatient centers may assist in reducing healthcare expenditure [5]. Still, the challenges associated with optimizing outcomes such as pain and satisfaction for TJA patients remain [6]. Despite best efforts, postoperative pain continues to pose a challenge for many surgical care teams, which may impede the transition to outpatient care $[7,8]$. Current pain management protocols have been extensively scrutinized to identify opportunities to maximize clinical outcomes and reduce postoperative complications [9-11]. However, the inclusion of opioid medications in such protocols has been associated with adverse outcomes that can be avoided [12].

Although opioids have classically been a mainstay in the management of postsurgical pain, cognizance of their unfavorable side-effect profile and potential to prolong hospital stays has spurred initiatives to identify 
alternative pain-control avenues [12-14]. A number of different agents and modalities have been studied as alternative pain therapies, including local or regional nerve blocks, acetaminophen, gabapentin, and nonsteroidal anti-inflammatory drugs (NSAIDs). In particular, NSAIDs have shown promise at maximizing analgesia while decreasing the opioid-related side effects and addiction risk [15-20]. Additionally, intravenous (IV) preparations have the benefit of greater bioavailability than oral medication for patients in need of acute pain management [21,22]. As opioid prescriptions are increasingly restricted and controlled, IV NSAID medications like ketorolac and diclofenac may be well suited to acutely decrease postoperative pain, increasing patient satisfaction, and facilitating earlier discharge in TJA patients [23,24].

Although several studies have evaluated the efficacy of IV ketorolac and diclofenac on postoperative outcomes and pain control, there is a paucity of literature comparing the two in the setting of primary TJA. As such, this study evaluated: 1) total opioid consumption; 2) postoperative pain intensity; 3) discharge disposition; 4) length of hospital stay; and 5) satisfaction with adjunctive IV diclofenac or ketorolac pain control in primary THA and TKA patients, at a single institution between 2017 and 2018.

\section{Materials And Methods \\ Patient selection}

Institutional Review Board approval was obtained for all aspects of this retrospective cohort study. All patients that underwent TJA with a single surgeon between March 1, 2017 and April 31, 2018 were identified and screened for study inclusion. Patients were included if they had undergone a primary TKA or THA and received adjunctive diclofenac or ketorolac as a part of their postoperative pain management regimen. Patients were excluded from the review if they were on methadone therapy, had a history of chronic opioid use, had a low calculated creatinine clearance (less than $30 \mathrm{~mL} / \mathrm{min} 2$ ), had undergone epidural anesthesia during their procedure, had a poor cardiac function, had an allergy to any NSAIDs, narcotics, or local anesthetics used in the study, or if they had undergone revision or conversion THA or TKA.

We identified a total of 136 eligible TJA patients who had received IV diclofenac or IV ketorolac that were blinded to their perioperative pain regimen. The final patient cohorts comprised 25 THA patients and 51 TKA patients who had received postoperative IV diclofenac, and 28 THA patients and 32 TKA patients who had received postoperative IV ketorolac.

\section{Pain regimen}

The standard pain regimen included: celecoxib $400 \mathrm{mg}$ per oral (PO) preoperatively, then celecoxib $200 \mathrm{mg}$ twice per day, starting on the evening of postoperative day one (POD1) (Table 1). 


\section{Cureus}

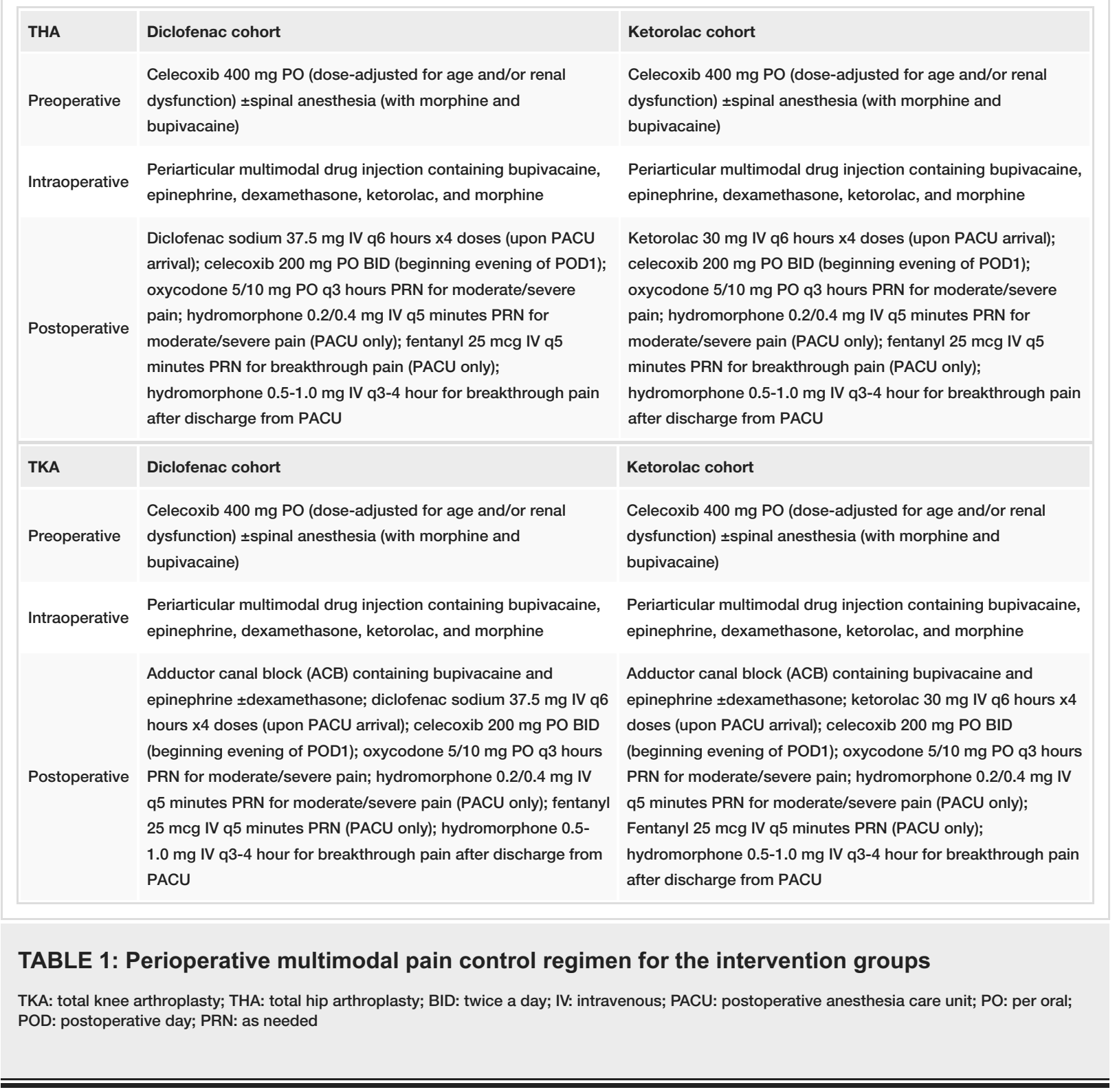

In addition, the diclofenac group received $37.5 \mathrm{mg}$ of IV diclofenac sodium every six hours for four doses, beginning on arrival to the postoperative anesthesia care unit (PACU). The ketorolac group similarly received $30 \mathrm{mg}$ of IV ketorolac every six hours for four doses upon arrival to the PACU. Oxycodone immediate-release ( 5 or $10 \mathrm{mg}$ PO) was made available postoperatively to all patients in both groups to ease breakthrough pain if necessary.

\section{Study variables}

Demographic information including gender, race, age, and body mass index (BMI) was collected for all patients. Opioid consumption [converted to morphine milligram equivalents [MME]), length of stay (LOS), visual analog scale (VAS) pain scores at eight-hour intervals, discharge destination, and post-admission satisfaction scores were collected to evaluate patient outcomes. Patient satisfaction with postoperative pain control was assessed via three questions selected from the Hospital Consumer Assessment of Healthcare Providers and Systems (HCAHPS) survey (Table 2) [25]. 


\section{Cureus}

Question

During this hospital stay, how well was your pain controlled?

During this hospital stay, how often was your pain well-controlled?

During this hospital stay, how often did the hospital staff do everything they could to

help you with your pain?
Answer

1: Never (least satisfied); 2: Sometimes; 3: Usually; 4: Always (most satisfied)

TABLE 2: Hospital Consumer Assessment of Healthcare Providers and Systems patient satisfaction questions

Pain intensity, expressed as area under the curve (AUC), was calculated utilizing VAS pain scores at eighthour intervals through the 48-hour postoperative period.

The THA patients in the IV diclofenac and IV ketorolac cohorts differed in race $(54.2$ vs. $82.1 \%$ white; $p$ : 0.029 ) and gender (33.3 vs. $60.7 \%$ female; p: 0.049); however, there were no differences in other demographic characteristics, including age (57.25 vs. 58.86 years; p: 0.641$)$ and BMI (30.66 vs. $28.81 \mathrm{~kg} / \mathrm{m} 2$; p: 0.297) (Table 3).

\begin{tabular}{|c|c|c|c|c|c|c|}
\hline & \multicolumn{3}{|l|}{ THA } & \multicolumn{3}{|l|}{ TKA } \\
\hline & Diclofenac & Ketorolac & P-value & Diclofenac & Ketorolac & P-value \\
\hline Total number & 25 & 28 & & 51 & 32 & \\
\hline \multicolumn{7}{|l|}{ Gender, n (\%) } \\
\hline Female & $8(33.3 \%)$ & $17(60.7 \%)$ & & $34(66.7 \%)$ & $20(62.5 \%)$ & \\
\hline Male & $16(66.7 \%)$ & $11(39.3 \%)$ & 0.049 & 17 (33.3\%) & 12 (37.5\%) & 0.698 \\
\hline \multicolumn{7}{|l|}{ Race, n (\%) } \\
\hline White & $13(54.2 \%)$ & $23(82.1 \%)$ & & 18 (35.3\%) & $17(53.1 \%)$ & \\
\hline Black & $11(45.8 \%)$ & $5(17.9 \%)$ & & $32(62.7 \%)$ & $15(46.9 \%)$ & \\
\hline Indian & $0(0 \%)$ & $0(0 \%)$ & 0.029 & $1(2.0 \%)$ & $0(0 \%)$ & 0.225 \\
\hline Mean age at surgery, years ( \pm SD) & $57.25( \pm 11.96)$ & $58.86( \pm 12.58)$ & 0.641 & $57.25( \pm 11.96)$ & $58.86( \pm 12.58)$ & 0.641 \\
\hline Mean BMI, kg/m², ( $\pm \mathrm{SD})$ & $30.66( \pm 7.34)$ & $28.81( \pm 5.27)$ & 0.297 & $34.5( \pm 6.11)$ & $33.3( \pm 6.86)$ & 0.430 \\
\hline Mean operative time, min, $( \pm S D)$ & $121.52( \pm 29.18)$ & $118.96( \pm 36.42)$ & 0.786 & $130( \pm 30.6)$ & $128( \pm 36.7)$ & 0.849 \\
\hline \multicolumn{7}{|l|}{ Anesthesia type, n (\%) } \\
\hline Spinal & $16(66.7 \%)$ & $24(85.7 \%)$ & & $2(3.9 \%)$ & $1(3.1 \%)$ & \\
\hline Spinal + general & $3(12.5 \%)$ & $1(3.6 \%)$ & & 41 (80.4\%) & 31 (96.9\%) & \\
\hline General & $5(20.8 \%)$ & 3 (10.7\%) & 0.245 & $8(15.7 \%)$ & $0(0 \%)$ & 0.059 \\
\hline
\end{tabular}

TABLE 3: Demographic characteristics in the IV diclofenac and IV ketorolac groups

THA: total hip arthroplasty; TKA: total knee arthroplasty; IV: intravenous; min: minute; n: number; SD: standard deviation

Baseline demographic characteristics including age, BMI, gender, race, and anesthesia type were similar between the two TKA groups ( $\mathrm{p}:>0.05$ for all).

\section{Statistical analysis}

Chi-square analysis/Fisher's exact test and Student's t-test were utilized to analyze categorical and continuous variables, respectively. A p-value of less than 0.05 was designated as statistically significant. All 


\section{Cureus}

statistical analysis was performed using IBM SPSS Statistics version 24 (IBM Corporation, Armonk, NY).

\section{Results}

For THA recipients, there was no significant difference between the IV diclofenac and ketorolac groups in mean total morphine milliequivalents (MME) opioid consumption at 12 (24.48 vs. $32.02 \mathrm{mg}$; p: 0.227 ) or 24 hours (17.71 vs. $18.25 \mathrm{mg}$; p: 0.915 ) postoperatively (Table 4).

\begin{tabular}{|c|c|c|c|}
\hline THA & Diclofenac & Ketorolac & P-value \\
\hline \multicolumn{4}{|c|}{ Mean opioid consumption, MME ( \pm SD) } \\
\hline MME 0-12 hours ( \pm SD) & $24.48( \pm 21.06)$ & $32.02( \pm 23.01)$ & 0.227 \\
\hline MME 12-24 hours (土SD) & $17.71( \pm 16.57)$ & $18.25( \pm 19.48)$ & 0.915 \\
\hline \multicolumn{4}{|l|}{ Mean pain intensity (AUC) } \\
\hline AUC 24 hours ( \pm SD) & $80.00( \pm 34.04)$ & $95.00( \pm 47.24)$ & 0.202 \\
\hline AUC 48 hours ( \pm SD) & $68.50( \pm 47.57)$ & $63.57( \pm 49.07)$ & 0.716 \\
\hline
\end{tabular}

\section{TABLE 4: Differences in pain control outcomes between IV diclofenac and IV ketorolac THA} patients

THA: total hip arthroplasty; MME: morphine milliequivalents; S.D.: standard deviation; AUC: area under the curve; IV: intravenous

There were also no differences found in postoperative pain intensity POD1 (80.00 vs. 95.00; p: 0.202) or POD2 (68.50 vs. 63.57 ; p: 0.716 ), LOS ( 2.00 vs. 2.18 days; p: 0.135 ), or discharge destination ( 87.5 vs. $89.3 \%$ home; p: 0.217) (Table 5). However, patients who received diclofenac were more satisfied with their pain control than those given ketorolac (Table 6).

\begin{tabular}{|c|c|c|c|}
\hline THA & Diclofenac & Ketorolac & P-value \\
\hline Mean length of stay, days ( \pm SD) & $2.00( \pm 0.417)$ & $2.18( \pm 1.79)$ & 0.135 \\
\hline \multicolumn{4}{|l|}{ Discharge destination, n (\%) } \\
\hline Home & $21(87.5 \%)$ & $25(89.3 \%)$ & \multirow{3}{*}{0.217} \\
\hline Inpatient rehab & $2(8.3 \%)$ & $0(0 \%)$ & \\
\hline Acute rehab & $1(4.2 \%)$ & $3(10.7 \%)$ & \\
\hline
\end{tabular}

\section{TABLE 5: Postoperative outcomes in IV diclofenac and IV ketorolac THA patients}

THA: total hip arthroplasty; SD: standard deviation; IV: intravenous 


\section{Cureus}

\begin{tabular}{|c|c|c|c|}
\hline THA & Diclofenac & Ketorolac & P-value \\
\hline \multicolumn{4}{|c|}{ Patient satisfaction question 1: “During this hospital stay, how well was your pain controlled?” } \\
\hline Always, n (\%) & $18(75.0 \%)$ & $12(48.0 \%)$ & \multirow{3}{*}{0.021} \\
\hline Usually, $n$ (\%) & $5(20.8 \%)$ & $4(16.0 \%)$ & \\
\hline Sometimes*, n (\%) & $1(4.2 \%)$ & $9(36.0 \%)$ & \\
\hline \multicolumn{4}{|c|}{ Patient satisfaction question 2: “During this hospital stay, how often was your pain well-controlled?” } \\
\hline Always ${ }^{\star}, \mathrm{n}(\%)$ & $19(79.2 \%)$ & $12(48.0 \%)$ & \multirow{3}{*}{0.019} \\
\hline Usually, n (\%) & $4(16.7 \%)$ & $4(16.0 \%)$ & \\
\hline Sometimes*, n (\%) & $1(4.2 \%)$ & $9(36.0 \%)$ & \\
\hline \multicolumn{4}{|c|}{ Patient Satisfaction Question 3: "During this hospital stay, how often did the hospital staff do everything they could to help you with your pain?" } \\
\hline Always ${ }^{\star}, \mathrm{n}(\%)$ & $23(95.8 \%)$ & $12(48.0 \%)$ & \multirow{3}{*}{0.001} \\
\hline Usually, n (\%) & $1(4.2 \%)$ & $4(16.0 \%)$ & \\
\hline Sometimes*, n (\%) & $0(0 \%)$ & $9(36.0 \%)$ & \\
\hline
\end{tabular}

\section{TABLE 6: Patient satisfaction scores in IV diclofenac and IV ketorolac THA patients}

THA: total hip arthroplasty; IV: intravenous

*Posthoc analysis (adjusted residuals)

Specifically, IV diclofenac recipients answered "Always" significantly more often than IV ketorolac patients in response to the questions "During this hospital stay, how well was your pain controlled?" (75.0 vs. $48.0 \%$; p: 0.021); "During this hospital stay, how often was your pain well-controlled?" (79.2 vs. 48.0\%; p: 0.019); and "During this hospital stay, how often did the hospital staff do everything they could to help you with your pain?” (95.8 vs. $48.0 \%$; p: 0.001).

For TKA recipients, patients in the IV diclofenac cohort had lower opioid consumption than patients in the IV ketorolac cohort during the first twelve hours postoperatively (21.6 vs. 32.8 MME; p: 0.037) (Table 7).

\begin{tabular}{|c|c|c|c|}
\hline TKA & Diclofenac & Ketorolac & P-value \\
\hline \multicolumn{4}{|c|}{ Mean opioid consumption, MME ( \pm SD) } \\
\hline MME 0-12 hours ( \pm SD) & $21.6( \pm 19.8)$ & $32.8( \pm 28.0)$ & 0.037 \\
\hline MME 12-24 hours ( \pm SD) & $19.0( \pm 19.7)$ & $21.5( \pm 21.8)$ & 0.596 \\
\hline \multicolumn{4}{|l|}{ Mean pain intensity (AUC) } \\
\hline AUC 24 hours ( $\pm S D$ ) & $81.6( \pm 43.8)$ & $93.6( \pm 49.1)$ & 0.251 \\
\hline AUC 48 hours ( \pm SD) & $85.5( \pm 48.0)$ & $84.3( \pm 51.3)$ & 0.911 \\
\hline
\end{tabular}

TABLE 7: Differences in pain control outcomes between IV diclofenac and IV ketorolac TKA patients

TKA: total knee arthroplasty; MME: morphine milliequivalents; SD: standard deviation; AUC: area under the curve; IV: intravenous

There were no differences between the diclofenac and ketorolac groups in opioid consumption at 24 hours (19.0 vs. 21.5 MME; p: 0.596), pain intensity on POD1 (81.6 vs. 93.6; p: 0.251 ) or POD2 (85.5 vs. 84.3; p: 0.911 ), or mean LOS ( 2.6 vs. 2.1 days; p: 0.063 ). The diclofenac group was less likely than the ketorolac group to be discharged home (58.8 vs. $84.4 \%$ home; p: 0.025 ) (Table 8 ). 


\section{Cureus}

\begin{tabular}{|c|c|c|c|}
\hline TKA & Diclofenac & Ketorolac & P-value \\
\hline Mean length of stay, days $( \pm \mathrm{SD})$ & $2.63( \pm 1.82)$ & $2.09( \pm 0.69)$ & 0.063 \\
\hline \multicolumn{4}{|l|}{ Discharge destination, n (\%) } \\
\hline Home $^{\star}$ & $30(58.8 \%)$ & $27(84.4 \%)$ & \multirow{4}{*}{0.025} \\
\hline Inpatient rehab* & $9(17.6 \%)$ & $0(0.0 \%)$ & \\
\hline Acute rehab & $8(15.7 \%)$ & $4(12.5 \%)$ & \\
\hline Subacute rehab & $4(7.8 \%)$ & $1(3.1 \%)$ & \\
\hline Difference in KSS score at 1 month, mean $( \pm S D)$ & $20.0( \pm 6.62)$ & $22.7( \pm 5.61)$ & 0.068 \\
\hline
\end{tabular}

\section{TABLE 8: Postoperative outcomes in IV diclofenac and IV ketorolac TKA patients}

TKA: total knee arthroplasty; SD: standard deviation; KSS: Knee Society Score; IV: intravenous

*Posthoc analysis (adjusted residuals)

There was no difference in mean postoperative Knee Society Score (KSS) between the groups (20.0 vs. 22.7; p: 0.068). Patients receiving diclofenac were significantly more satisfied with their pain control experience than those who received ketorolac (Table 9).

\begin{tabular}{|c|c|c|c|}
\hline TKA & Diclofenac & Ketorolac & P-value \\
\hline \multicolumn{4}{|c|}{ Patient satisfaction question 1: “During this hospital stay, how well was your pain controlled?" } \\
\hline Always*, n (\%) & $37(74.0)$ & $12(46.2 \%)$ & \multirow{3}{*}{$<0.001$} \\
\hline Usually, n (\%) & $13(26.0 \%)$ & $5(19.2 \%)$ & \\
\hline Sometimes*, n (\%) & $0(0 \%)$ & $9(34.6 \%)$ & \\
\hline \multicolumn{4}{|c|}{ Patient satisfaction question 2: “During this hospital stay, how often was your pain well-controlled?" } \\
\hline Always*, n (\%) & $35(70.0 \%)$ & $12(46.2 \%)$ & \multirow{3}{*}{$<0.001$} \\
\hline Usually, n (\%) & $15(30 \%)$ & $5(19.2 \%)$ & \\
\hline Sometimes*, n (\%) & $0(0 \%)^{\star}$ & $9(34.6 \%)^{*}$ & \\
\hline \multicolumn{4}{|c|}{ Patient satisfaction question 3: "During this hospital stay, how often did the hospital staff do everything they could to help you with your pain?" } \\
\hline Always*, n (\%) & $48(96.0 \%)$ & $12(46.2 \%)$ & \multirow{3}{*}{$<0.001$} \\
\hline Usually, n (\%) & $2(4.0)$ & $6(23.1 \%)$ & \\
\hline Sometimes*, n (\%) & $0(8 \%)$ & $8(30.8 \%)$ & \\
\hline
\end{tabular}

\section{TABLE 9: Patient satisfaction scores in IV diclofenac and IV ketorolac TKA patients}

TKA: total knee arthroplasty; IV: intravenous

*Posthoc analysis (adjusted residuals)

Specifically, IV diclofenac recipients answered "Always" more often than IV ketorolac patients in response to the questions "During this hospital stay, how well was your pain controlled?" (74.0 vs. $46.2 \%$; p: $<0.001$ ); "During this hospital stay, how often was your pain well-controlled?" (70.0 vs. $46.2 \%$; p: $<0.001$ ); and "During this hospital stay, how often did the hospital staff do everything they could to help you with your pain?” (96.0 vs. $46.2 \%$; p: <0.001).

\section{Discussion}


Optimizing pain control regimens following TJA is an important aspect to help these procedures transition to the outpatient setting, as improved pain management can improve clinical outcomes and curtail costs [26]. Due to their efficacy in pain relief and in reducing opioid consumption, NSAIDs such as diclofenac and ketorolac have emerged as a promising adjunctive pain control modalities $[16,20]$. The present study demonstrated that while primary TKA patients receiving IV diclofenac had significantly lower opioid consumption during the first 12 hours postoperatively, there was no difference in opioid consumption at 24 hours when compared to those who received IV ketorolac. In addition, there were no significant differences in opioid consumption or discharge destination in THA patients and no differences in postoperative pain intensity in both TKA and THA patients. However, TKA and THA patients on the IV diclofenac regimen were significantly more satisfied with their postoperative pain experience than those on the IV ketorolac regimen. The IV formulation of both these nonopioid medications appears effective in managing postoperative TJA pain and may be useful in allowing patients to have a faster discharge.

There are some limitations to this study. As we only included procedures performed by a single surgeon at a single institution, the sample sizes are small. However, this was meant to be a preliminary study, reporting the early outcomes of IV NSAIDs in TJA. Additionally, data input and collection may have been susceptible to human error. To minimize this risk, data collection was limited to one investigator and the same nursing floor. Finally, satisfaction was significantly higher for the diclofenac group, despite similar outcomes. This could be due to the small sample sizes, as patients were blinded, and pain intensity was similar in all groups. Nevertheless, the present study is a great starting point for the investigation of TJA outcomes in patients receiving adjunctive IV diclofenac and IV ketorolac.

Although TKA patients receiving IV diclofenac had lower opioid consumption at 12 hours, our findings revealed no difference in 24-hour opioid consumption for TKA and THA patients in the IV diclofenac and IV ketorolac groups. Other studies have reported on the comparative decrease in opioid consumption with both IV diclofenac and IV ketorolac. A double-blind, randomized control trial by Alexander et al. compared morphine consumption, as well as pain scores and opioid side effects, in 102 TKA and THA patients who were given IV diclofenac, IV ketorolac, or placebo preoperatively [27]. They revealed that diclofenac and ketorolac patients both consumed significantly less morphine than the placebo group (p: 0.032), and experienced significantly less opioid side effects ( $\mathrm{p}:<0.05$ ). In addition, George et al. compared adjunctive IV diclofenac to standard pain management in a retrospective study of 113 THA patients, finding a significant reduction in opioid consumption on POD1 and 2 for IV diclofenac patients (p: 0.001) [24]. Furthermore, another study of 52 TKAs by George et al. assessed the addition of IV diclofenac to a multimodal pain control regimen and demonstrated a significant reduction in opioid consumption among patients who received IV diclofenac (p: 0.041) [23]. These results show the efficacy of IV NSAIDs in reducing the need for postoperative opioids, an important goal in the current fight to minimize opioid misuse and potential addiction.

The present study demonstrated no differences in postoperative pain intensity or LOS between the IV diclofenac and IV ketorolac THA and TKA cohorts. Although prior studies have found decreased postoperative pain with the use of ketorolac and diclofenac in TJA, no study has demonstrated a significant difference between the two drugs. Etches et al. performed a multicenter, double-blinded, randomized controlled trial (RCT) comparing postoperative pain control between TKA and THA patients who received general anesthesia with placebo $(n=88)$ or with continuous infusion ketorolac $(n=86)$ [28]. Compared to placebo, the ketorolac cohort had significantly decreased pain intensity at two, four, and six hours postoperatively (p: $<0.05$ for all). A double-blind study by Minotti et al. demonstrated similar results, finding that in 180 patients with cancer pain, $10 \mathrm{mg}$ of intramuscular ketorolac was equivalent in analgesic efficacy to $75 \mathrm{mg}$ of intramuscular diclofenac [29]. Moreover, a prospective, double-blinded comparative study by Kostamovaara et al. randomized primary THA patients $(n=85)$ to receive ketorolac $(n=28)$, diclofenac $(n=$ $28)$, or ketoprofen $(n=29)[20]$. The authors reported no difference between groups in median VAS scores up through sixteen hours postoperatively ( $\mathrm{p}:>0.05$ for all time points). These studies all corroborate our results and reinforce the ability of NSAIDs, particularly diclofenac and ketorolac, to provide adequate analgesia and have comparable LOS. These medications also appear well suited for utilization in the outpatient setting, as they are most efficacious in the first 24 hours after surgery.

Although patients in both THA and TKA diclofenac cohorts had increased satisfaction with IV diclofenac over IV ketorolac, outcomes were similar between the cohorts. Similar postoperative pain intensities and opioid consumption demonstrate the effectiveness of both drugs in managing postoperative pain. However, there are no studies comparing the cost of IV diclofenac to IV ketorolac for orthopedic surgery. In our institution, there is a vast pricing difference between IV diclofenac and IV ketorolac. The cost of four doses of IV diclofenac is USD 48.00, while the cost of four doses of IV ketorolac is USD 3.12. When extrapolated for 1,000 patients, the cost savings between these two drugs becomes apparent (USD 44,880 saved per 1,000 patients). This supports the utilization of IV ketorolac in postoperative pain management to yield the greatest cost savings. Nonetheless, the utilization of IV NSAIDs for pain management after TJA appears beneficial in reducing opioid consumption and may be well suited to supporting outpatient surgery in the future.

\section{Conclusions}


Optimizing postoperative pain management is essential to improving clinical outcomes and reducing costs, especially as TJA moves out of the hospital. The addition of NSAIDs to the postoperative pain regimen is a promising way of decreasing opioid consumption and reducing postoperative pain. This study demonstrated that both TKA and THA patients treated with IV diclofenac had no difference in postoperative pain intensity while THA patients had no difference in opioid consumption relative to those treated with IV ketorolac. Further comparison of IV NSAIDs with other IV pain medications may provide broader insight into the ideal management of postoperative pain for this widening patient population.

\section{Additional Information \\ Disclosures}

Human subjects: Consent was obtained by all participants in this study. LifeBridge Health Institutional Review Board issued approval NA. LifeBridge Health IRB has determined that according to applicable federal regulations (45 CFR 46.101) this project is exempt from IRB review as this does not meet the criteria for human subjects research. Animal subjects: All authors have confirmed that this study did not involve animal subjects or tissue. Conflicts of interest: In compliance with the ICMJE uniform disclosure form, all authors declare the following: Payment/services info: All authors have declared that no financial support was received from any organization for the submitted work. Financial relationships: Ronald E Delanois declare(s) Research Support from Orthofix, Inc. Ronald E Delanois declare(s) Research Support from Stryker. Ronald E Delanois declare(s) Research Support from United Orthopedics. Ronald E Delanois declare(s) Research Support from Flexion Therapeutics. Ronald E Delanois declare(s) Research Support from TissueGene. Ronald E Delanois declare(s) Board or committee member from Baltimore City Medical Society. Other relationships: All authors have declared that there are no other relationships or activities that could appear to have influenced the submitted work.

\section{References}

1. Center for Medicare \& Medicaid Services: Comprehensive Care for Joint Replacement Model . (2020). Accessed: January 7, 2020: https://innovation.cms.gov/initiatives/cjr.

2. Stambough JB, Nunley RM, Curry MC, Steger-May K, Clohisy JC: Rapid recovery protocols for primary total hip arthroplasty can safely reduce length of stay without increasing readmissions. J Arthroplasty. 2015, 30:521-6. 10.1016/j.arth.2015.01.023

3. Edwards PK, Kee JR, Mears SC, Barnes CL: Is rapid recovery hip and knee replacement possible and safe in the octogenarian patient?. J Arthroplasty. 2018, 33:316-9. 10.1016/j.arth.2017.09.060

4. CY 2020 Medicare Hospital Outpatient Prospective Payment System and Ambulatory Surgical Center Payment System Final Rule (CMS-1717-FC). (2020). Accessed: January 7, 2020: https://www.cms.gov/newsroom/fact-sheets/cy-2020-medicare-hospital-outpatient-prospective-paymentsystem-and-ambulato....

5. Kurtz S, Ong K, Lau E, Mowat F, Halpern M: Projections of primary and revision hip and knee arthroplasty in the United States from 2005 to 2030. J Bone Joint Surg Am. 2007, 89:780-5. 10.2106/JBJS.F.00222

6. Gaffney CJ, Pelt CE, Gililland JM, Peters CL: Perioperative pain management in hip and knee arthroplasty . Orthop Clin North Am. 2017, 48:407-19. 10.1016/j.ocl.2017.05.001

7. Grosu I, Thienpont E, De Kock M, Scholtes JL, Lavand'homme P: Dynamic view of postoperative pain evolution after total knee arthroplasty: a prospective observational study. Minerva Anestesiol. 2016, 82:27483.

8. Danoff JR, Goel R, Sutton R, Maltenfort MG, Austin MS: How much pain is significant? Defining the minimal clinically important difference for the visual analog scale for pain after total joint arthroplasty. J Arthroplasty. 2018, 33:S71-5. 10.1016/j.arth.2018.02.029

9. Etcheson JI, Gwam CU, George NE, Virani S, Mont MA, Delanois RE: Opioids consumed in the immediate post-operative period do not influence how patients rate their experience of care after total hip arthroplasty. J Arthroplasty. 2018, 33:1008-11. 10.1016/j.arth.2017.10.033

10. Gwam CU, Mohamed N, Mistry JB, et al.: Pain management with adductor canal blockade or multimodal periarticular analgesia in elderly total knee arthroplasty patients. Surg Technol Int. 2017, 30:352-8.

11. Argoff CE: Recent management advances in acute postoperative pain . Pain Pract. 2014, 14:477-87. 10.1111/papr.12108

12. Wheeler M, Oderda GM, Ashburn MA, Lipman AG: Adverse events associated with postoperative opioid analgesia: a systematic review. J Pain. 2002, 3:159-80. 10.1054/jpai.2002.123652

13. Morrone LA, Scuteri D, Rombolà L, Mizoguchi H, Bagetta G: Opioids resistance in chronic pain management. Curr Neuropharmacol. 2017, 15:444-56. 10.2174/1570159X14666161101092822

14. Levin P: The opioid epidemic: impact on orthopaedic surgery . J Am Acad Orthop Surg. 2015, 23:e36-7. 10.5435/JAAOS-D-15-00250

15. Andersen K V, Nikolajsen L, Haraldsted V, Odgaard A, Søballe K: Local infiltration analgesia for total knee arthroplasty: should ketorolac be added?. Br J Anaesth. 2013, 111:242-8. 10.1093/bja/aet030

16. Schwinghammer AJ, Isaacs AN, Benner RW, Freeman H, O'Sullivan JA, Nisly SA: Continuous infusion ketorolac for postoperative analgesia following unilateral total knee arthroplasty. Ann Pharmacother. 2017, 51:451-6. 10.1177/1060028017694655

17. Gombotz H, Lochner R, Sigl R, Blasl J, Herzer G, Trimmel H: Opiate sparing effect of fixed combination of diclophenac and orphenadrine after unilateral total hip arthroplasty: a double-blind, randomized, placebocontrolled, multi-centre clinical trial. Wien Med Wochenschr. 2010, 160:526-34. 10.1007/s10354-010-0829-7

18. Silvanto M, Lappi M, Rosenberg PH: Comparison of the opioid-sparing efficacy of diclofenac and ketoprofen for 3 days after knee arthroplasty. Acta Anaesthesiol Scand. 2002, 46:322-8. 10.1034/j.1399-6576.2002.t011-460316.x 
19. Gora-Harper ML, Record KE, Darkow T, Tibbs PA: Opioid analgesics versus ketorolac in spine and joint procedures: impact on healthcare resources. Ann Pharmacother. 2001, 35:1320-6. 10.1345/aph.10340

20. Kostamovaara PA, Hendolin H, Kokki H, Nuutinen LS: Ketorolac, diclofenac and ketoprofen are equally efficacious for pain relief after total hip replacement surgery. Br J Anaesth. 1998, 81:369-72. 10.1093/bja/81.3.369

21. Claeys MA, Camu F, Maes V: Prophylactic diclofenac infusions in major orthopedic surgery: effects on analgesia and acute phase proteins. Acta Anaesthesiol Scand. 1992, 36:270-5. 10.1111/j.13996576.1992.tb03463.x

22. van der Westhuizen J, Kuo PY, Reed PW, Holder K: Randomised controlled trial comparing oral and intravenous paracetamol (acetaminophen) plasma levels when given as preoperative analgesia. Anaesth Intensive Care. 2011, 39:242-6. 10.1177/0310057X1103900214

23. George NE, Gurk-Turner C, Etcheson JI, et al.: The addition of diclofenac to a multimodal pain control regimen decreases postoperative pain and opioid consumption. Surg Technol Int. 2017, 31:346-51.

24. George NE, Gurk-Turner C, Castrodad IMD, et al.: Adjunctive intravenous diclofenac decreases opioid consumption and increases satisfaction in the primary total hip arthroplasty population. Surg Technol Int. 2019, 34:456-61.

25. HCAHPS: patients' perspectives of care survey. (2019). Accessed: January 7, 2020: https://www.cms.gov/Medicare/Quality-Initiatives-Patient-AssessmentInstruments/HospitalQualityInits/HospitalHCAHPS.html.

26. Kim K, Elbuluk A, Yu S, Iorio R: Cost-effective peri-operative pain management: assuring a happy patient after total knee arthroplasty. Bone Joint J. 2018, 100:55-61. 10.1302/0301-620X.100B1.BJJ-2017-0549.R1

27. Alexander R, El-Moalem HE, Gan TJ: Comparison of the morphine-sparing effects of diclofenac sodium and ketorolac tromethamine after major orthopedic surgery. J Clin Anesth. 2002, 14:187-92. 10.1016/s09528180(01)00382-8

28. Etches RC, Warriner CB, Badner N, et al.: Continuous intravenous administration of ketorolac reduces pain and morphine consumption after total hip or knee arthroplasty. Anesth Analg. 1995, 81:1175-80. 10.1097/00000539-199512000-00010

29. Minotti V, Betti M, Ciccarese G, Fumi G, Tonato M, Del Favero A: A double-blind study comparing two single-dose regimens of ketorolac with diclofenac in pain due to cancer. Pharmacotherapy. 1998, 18:504-8. 10.1002/j.1875-9114.1998.tb03113.x 